\title{
Ecology and conservation of threatened plants in Tapkeshwari Hill ranges in the Kachchh Island, Gujarat, India
}

\author{
P.N. Joshi ${ }^{1}$, Ekta B. Joshi ${ }^{2} \&$ B.K. Jain ${ }^{3}$ \\ ${ }^{1}$ Sahjeevan, 175-Jalaram Society, Vijay Nagar, Bhuj, Kachchh, Gujarat 370001, India \\ ${ }^{2}$ Matruchhaya Kanya Vidhyalay, Matruchhaya Road, Bhuj, Kachchh, Gujarat 370001, India \\ ${ }^{3}$ M.G. Science Institute, Gujarat Uiniversity, Ahmedabad, Gujarat, India \\ Email: joshi_pn@yahoo.com (corresponding author),noopur_pj@yahoo.co.in, bkjain_mgsc@yahoo.com
}

\section{Date of publication (online): 26 February 2012 Date of publication (print): 26 February 2012 ISSN $0974-7907$ (online) | 0974-7893 (print) \\ Editor: N.P. Balakrishnan \\ Manuscript details: \\ Ms \# 02410 \\ Received 23 February 2010 \\ Final received 03 November 2011 \\ Finally accepted 24 January 2012}

Citation: Joshi, P.N., E.B. Joshi \& B.K Jain (2012). Ecology and conservation of threatened plants in Tapkeshwari Hill ranges in the Kachch Island, Gujarat, India. Journal of Threatened Taxa 4(2): 2390-2397.

Copyright: (c) P.N. Joshi, Ekta B. Joshi \& B.K. Jain 2012. Creative Commons Attribution 3.0 Unported License. JoTT allows unrestricted use of this article in any medium for non-profit purposes, reproduction and distribution by providing adequate credit to the authors and the source of publication.

Author Details: See end of this article.

Author Contribution: All author contributed in the study as well as in the current paper

Acknowledgements: Shri. Maneklal ShahDada, Trustee; Mrs. Premlataben Nehlani, Principal and Mrs. Jyotiben Chandwani, ExPrincipal, Matruchhaya Kanya Vidhyalay, Station Road, Bhuj were the constant source of encouragement and support. We thank them for proving all facilities in the School. We would like to thank Mr. R.L. Meena, IFS, Conservator of Forests, Kachchh Circle; Mr. L.N. Jadeja (Former DCF-West), Mr. D.T. Vasavada, (DCFWest), Mr. H.P. Waria (ACF) and Mr. M.B. Pate (RFO) (Kachchh West Division), Gujarat State Forest Department (GSFD), Bhuj for giving permission to work in the Tapkeshwari Hill Range Forests.

\begin{abstract}
The survey was conducted in Tapkeshwari Hill Range (THR) areas, wherever threatened plant species were said to exist, based on secondary information in literature. Thirteen plant species categorized as 'Threatened' by the World Conservation Monitoring centre (WCMC 1994) and also listed under various threat categories in the Red Data Book of Indian Plants (Nayar \& Sastry 1988) were surveyed in the THR. All the RET plants reported from the study area occupied eight major habitat types. Thorn mixed forests harbored the highest number of individuals (560) of all RET plants, followed by open scrubs (345 individuals), Acacia senegal forests (328) and thorn mixed scrubs (293). Field observations showed that except Helichrysum cutchicum, all the other RET plant species were reported with very low seedlings and regeneration ratio. This paper discusses the status, distribution and threats faced and the conservation implications at border regions of some of the threatened plants of the arid Kachchh district.
\end{abstract}

Keywords: Conservation, distribution, ecology, endangered, rare, threatened, threats.

\section{INTRODUCTION}

Zietsman et al. (2008) stated that small and isolated populations often suffer from disrupted biological interactions. Nearly 1500 species of higher plants in India are listed as threatened, most of which are angiosperms (Daniels \& Jayanthi 1996). These plants have their own ecological role in the ecosystem and therefore, the conservation status of lesser known plant species and isolated populations need to be assessed both within individual populations and at the metapopulation level (Shaw \& Burns 1997). There is reported work in the past in Tapkeshwar Hill Range (THR) on threatened species, especially their ecological requirements. This study is intended to highlight the status and distribution of the species in the study area, the ecological characteristics necessary for their survival, and the threats faced by some of the species designated by following the criteria devised by WCMC and IUCN (Nayar \& Sastry 1988; WCMC 1994; Bhandari et al. 1996; GES et al. 2002).

\section{MATERIALS AND METHODS}

The study area (Tapkeshwari Hill Range - THR) of more than $140 \mathrm{~km}^{2}$ (14,400ha) covering nine villages under two taluks, i.e. Bhuj and Mundra was surveyed (Image 1). THR is the largest unexplored hilly tract in the district. It is close to Bhuj City, the district headquarters $(7 \mathrm{~km})$ and provides a high diversity of floral species in various vegetation types or 
habitats like Euphorbia scrubs, Prosopis scrubs, thorn mixed scrubs, open scrubs, thorn mixed forests with Acacia senegal, A. nilotica and Salvadora mixed (Image 2). Considering the high floral diversity and unique vegetation assemblage of this range, it has been suggested that, this tract and adjoining sites may be declared as Ecologically Sensitive Areas (ESA) (Joshi 2002).

The selected hill ranges experience extremes of weather condition and have three seasons, consisting of winter, summer and monsoon-winter (November to February; minimum averaging $\left.10^{\circ} \mathrm{C}\right)$, summer (March to June; maximum $38.7^{\circ} \mathrm{C}$ ) and monsoon (July to September; average $394.7 \mathrm{~mm}$ in $2007-2009$ and for 16.2 days).

The survey was conducted in the study areas wherever rare, endangered and threatened (RET) plant taxa were said to exist, based on information in the literature (Nayar \& Sastry 1988; WCMC 1994; GES et al. 2002). In addition, other adjoining areas, which had similar habitat types where the plants were seen during the survey, were also searched. A combination of belt transects with centred quadrates method were used for sampling. Belt transects of $5 \mathrm{~m}$ width and length

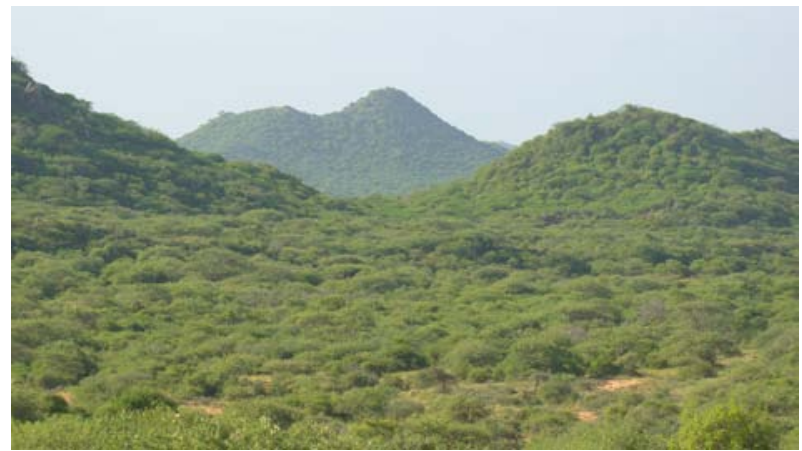

Image 2. Overall distribution of RET plants in various habitats

extending to the entire width of the patch were laid. Within this belt, species specific search was carried out and once a target species was located, a speciescentred circular plot of $5 \mathrm{~m}$ radius in the case of shrubs and 1 to $2 \mathrm{~m}$ radius in the case of herbs were laid. In case of abundance of plants, belt transects radiating from the edge of the aquatic body in eight directions were laid to assess the number, and the extent of their spread from the main microhabitat was used to record all other parameters as above.
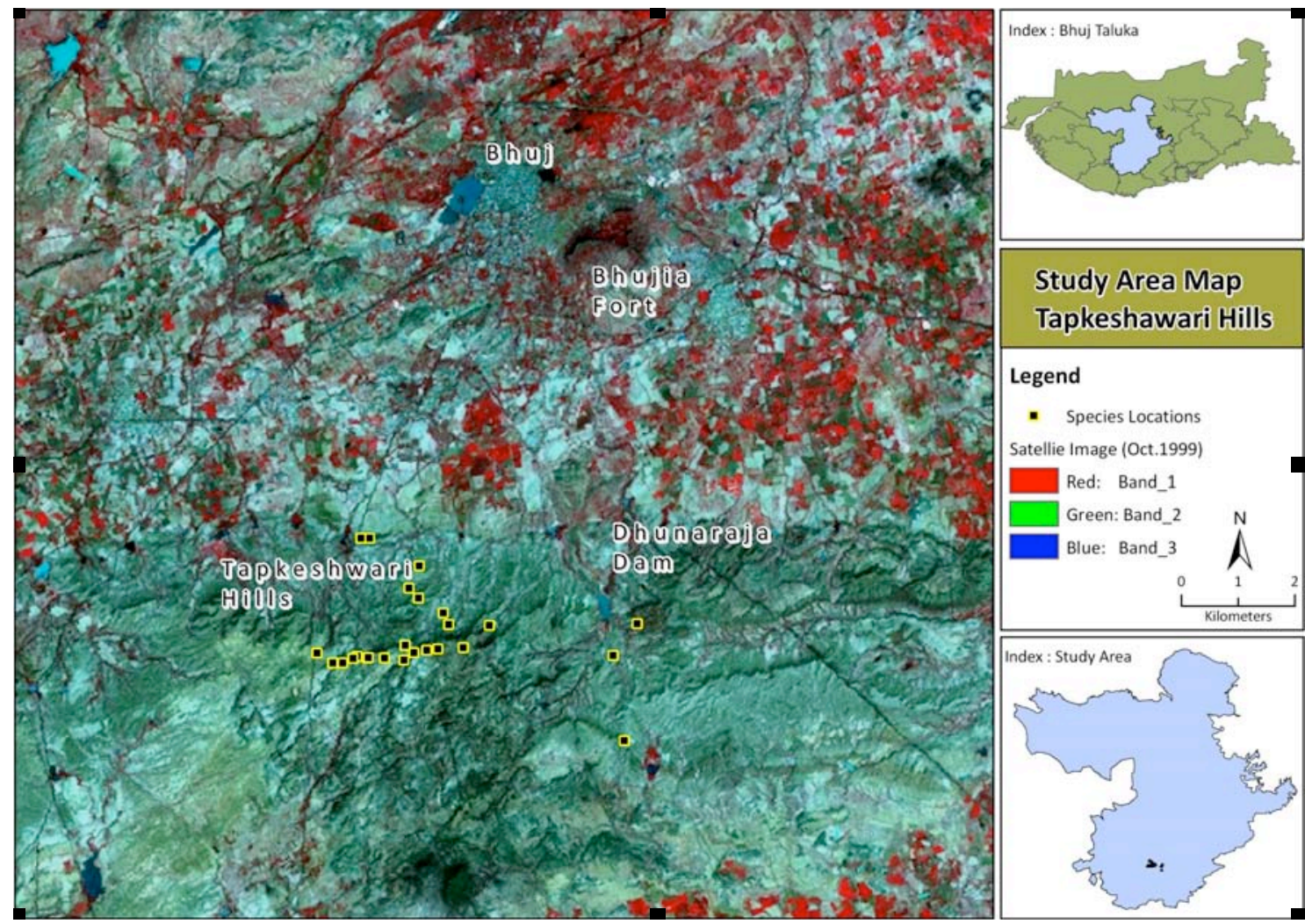

Image 1. The location of study area 


\section{RESULTS}

\section{IUCN- RET Plants Reports}

Thirteen plant species categorized as threatened by the World Conservation Monitoring Centre (WCMC 1994) and also listed under various threat categories in the Red Data Book of Indian Plants (Nayar \& Sastry 1988) were surveyed in the THR. In many rare species classifications, including the Red Book listings of the IUCN, two types of rarity — natural and induced - are not always clearly distinguished. Some species that are naturally rare are also ranked as threatened with extinction. While naturally rare species can be more vulnerable to extinction than common ones, rarity in itself is not synonymous with extinction threat. Understanding the difference between natural and induced rarity is important for focusing conservation efforts.

Out of the 19 RET plants recorded so far from Kachchh (Shah 1978; Nayar \& Sastry 1988; Raole 1993; WCMC 1994; GES et al. 2002), 13 taxa were located in the study area: six herbs, four undershrubs, two shrubs and one climber. Among these, Dipcadi erythraeum, Dactyliandra welwitschii, Indigofera caerulea var. monosperma and Pavonia ceratocarpa had very low numbers, i.e. 9, 13, 16 and 19 individuals, respectively, and had highly restricted distribution in THR. Commiphora wightii, Ipomoea kotschyana, Helichrysum cutchicum and Campylanthus ramosissimus showed wider distribution and had 612, 440, 245 and 235 individuals, respectively (Table 1). The details on abundance, habitats and threats of each taxon with their present status mentioned by different authorities are given in Table 1.

\section{Distribution and age structure status}

Overall distribution status of RET taxa in the study area: All the RET plants reported from the study area occupied eight major habitat types, of which thorn mixed scrub, open scrub and Acacia senegal forest harbored the highest number (10 in each) of taxa. The second highest number of taxa (9) was recorded from thorn mixed forest and Euphorbia scrub and so on (Table 2). Interestingly, thorns mixed forest harbored the highest number of individuals (560) of all RET plants, followed by open scrub (345 individuals), Acacia senegal forest (328), thorn mixed scrub (293) and so on (Fig. 1).
Campylanthus ramosissimus, Ipomoea kotschyana and Pavonia ceratocarpa were restricted to a single favorable habitat, viz., open scrub, thorn mixed forest and Euphorbia scrub respectively. Commiphora wightii, Convolvulus stocksii, Ephedra foliata and Helichrysum cutchicum also showed more affinity to the thorn mixed scrub (31.37\%), thorn mixed forest (44.88\%), thorn mixed forest (45.45\%) and Acacia senegal forest $(30.20 \%)$ respectively (Table 2 ).

Figure 1 shows there is no co-relation between the total number of plots (laid down for sampling) and individual count of RET plants in each habitat in the study area.

Age structure status of RET plants in THR: In this title detailed study on the RET plant species reproduction (with different age classes), regeneration, recruitment and adult plants were documented. However, a total of 13 species have been reported as threatened species in the study area. Only four species like Campylanthus ramosissimus, Citrullus colocynthis, Commiphora wightii and Helichrysum cutchicum were recorded under various reproduction classes in the sample area (Table 3).

Quantification of the reproductive stage of annual herbaceous plants is difficult when compared to bushy perennials because of their smaller size and very short life spans. It is further complicated if it has restricted distribution and low abundance. Within the sample area, field observation showed that except Helichrysum cutchicum, all other RET plant species are reported with very low seedlings and regeneration ratio (Table 3 ) when compared with the adult plants. In addition, low abundance of some RET plant species could be inherent and for others it may be failure of regeneration.

\section{Threats faced}

The details of the different kinds of threats faced by the RET plants species were also reported with respect to different stresses and the total number of plants affected along with information on each threat. Species-wise natural and anthropogenic threats faced and the individuals affected are given in Table 4. Natural and anthropogenic disturbances can have dramatic consequences for population growth, particularly for small populations of threatened plants (Coates et al. 2006; Tian et al. 2007); a plant species might be naturally rare because its habitat is restricted 
Table 1. Ecology and distribution of RET plants in various habitats

\begin{tabular}{|c|c|c|c|c|c|c|c|}
\hline Species & Family & No & $\begin{array}{c}\text { Preferable } \\
\text { habitat }\end{array}$ & Threats & Remarks & Status & Source \\
\hline $\begin{array}{l}\text { Campylanthus } \\
\text { ramosissimus Wt. }\end{array}$ & Scrophulariaceae & 235 & Open scrub & Habitat loss & $\begin{array}{l}\text { Restricted to certain } \\
\text { sandstone formations and } \\
\text { lime stone hills }\end{array}$ & $\begin{array}{l}\text { Rare } \\
\text { Endangered }\end{array}$ & $\begin{array}{l}\text { Nayar \& Sastry } 1988 \\
\text { WCMC } 1994 \\
\text { CREB-GUIDE } 2002\end{array}$ \\
\hline $\begin{array}{l}\text { Citrullus colocynthis } \\
\text { (L.) Soland. }\end{array}$ & Cucurbitaceae & 65 & $\begin{array}{l}\text { Thorn mixed } \\
\text { forest }\end{array}$ & Habitat loss & $\begin{array}{l}\text { Recorded on sand dunes } \\
\text { and sandy grounds }\end{array}$ & $\begin{array}{l}\text { Rare } \\
\text { Common }\end{array}$ & $\begin{array}{l}\text { Bhandari } 1990 \\
\text { CREB-GUIDE } 2002\end{array}$ \\
\hline $\begin{array}{l}\text { Commiphora wightii } \\
\text { (Arnott) Bhandari }\end{array}$ & Burseraceae & 612 & $\begin{array}{l}\text { Thorn mixed } \\
\text { scrub }\end{array}$ & $\begin{array}{l}\text { Over } \\
\text { exploitation }\end{array}$ & $\begin{array}{l}\text { Dominant on rocky hillocks } \\
\text { and hard gravelly soils }\end{array}$ & $\begin{array}{l}\text { Threatened } \\
\text { Not Evaluated } \\
\text { Indeterminate } \\
\text { Common }\end{array}$ & $\begin{array}{l}\text { Rao } 1981 \\
\text { IUCN } \\
\text { WCMC } 1994 \\
\text { CREB-GUIDE } 2002\end{array}$ \\
\hline $\begin{array}{l}\text { Convolvulus stocksii } \\
\text { Boiss. }\end{array}$ & Convolvulaceae & 127 & $\begin{array}{l}\text { Thorn mixed } \\
\text { forest }\end{array}$ & $\begin{array}{l}\text { Over } \\
\text { grazing }\end{array}$ & $\begin{array}{l}\text { Mostly restricted to loamy } \\
\text { and gravelly soils with } \\
\text { moderate soil depth }\end{array}$ & \begin{tabular}{|l|} 
Threatened \\
Rare \\
Indeterminate \\
Common \\
\end{tabular} & $\begin{array}{l}\text { Rao } 1981 \\
\text { Sabnis \& Rao } 1983 \\
\text { WCMC } 1994 \\
\text { CREB-GUIDE } 2002 \\
\end{array}$ \\
\hline $\begin{array}{l}\text { Dactyliandra } \\
\text { welwitschii Hook.f. }\end{array}$ & Cucurbitaceae & 13 & $\begin{array}{l}\text { Thorn mixed } \\
\text { forest }\end{array}$ & - & $\begin{array}{l}\text { Mostly associated with } \\
\text { Euphorbia cactus }\end{array}$ & $\begin{array}{l}\text { Common } \\
\text { Data Deficient }\end{array}$ & $\begin{array}{l}\text { Bhandari } 1990 \\
\text { CREB-GUIDE } 2002\end{array}$ \\
\hline $\begin{array}{l}\text { Dipcadi erythraeum } \\
\text { Webb. \& Berth. }\end{array}$ & Liliaceae & 9 & Open scrub & Erosion & $\begin{array}{l}\text { Site specific and require } \\
\text { moist soil substratum }\end{array}$ & $\begin{array}{l}\text { Rare } \\
\text { Indeterminate } \\
\text { Common }\end{array}$ & $\begin{array}{l}\text { Rao } 1981 \\
\text { WCMC } 1994 \\
\text { CREB-GUIDE } 2002\end{array}$ \\
\hline $\begin{array}{l}{ }^{*} \text { Ephedra foliata } \\
\text { Boiss. \& Kot.ex Boiss }\end{array}$ & Ephedraceae & 55 & $\begin{array}{l}\text { Thorn mixed } \\
\text { forest }\end{array}$ & $\begin{array}{l}\text { Cutting } \\
\text { associated } \\
\text { plants }\end{array}$ & $\begin{array}{l}\text { Mainly found on sandy, } \\
\text { gravelly or even rocky } \\
\text { areas }\end{array}$ & $\begin{array}{l}\text { Rare } \\
\text { Not Evaluated } \\
\text { Vulnerable }\end{array}$ & $\begin{array}{l}\text { Rao } 1981 \\
\text { IUCN } \\
\text { CREB-GUIDE } 2002\end{array}$ \\
\hline $\begin{array}{l}\text { Helichrysum } \\
\text { cutchicum (C.B. Cl.) } \\
\text { Rolla Rao et Des. }\end{array}$ & Asteraceae & 245 & $\begin{array}{l}\text { Acacia } \\
\text { senegal } \\
\text { forest }\end{array}$ & $\begin{array}{l}\text { Habitat } \\
\text { degradation }\end{array}$ & $\begin{array}{l}\text { Prefer undulating terrain } \\
\text { and sand stone with } \\
\text { sparse grasses cover }\end{array}$ & $\begin{array}{l}\text { Rare } \\
\text { Rare \& } \\
\text { Endemic } \\
\text { Lower Rick }\end{array}$ & $\begin{array}{l}\text { Nayar \& Sastry } 1988 \\
\text { WCMC } 1994 \\
\text { Sabnis \& Rao } 1983 \\
\text { CREB-GUIDE } 2002\end{array}$ \\
\hline $\begin{array}{l}\text { Indigofera caerulea } \\
\text { Roxb. var. } \\
\text { monosperma (Sant.) } \\
\text { Sant. }\end{array}$ & Fabaceae & 16 & Open scrub & $\begin{array}{l}\text { Over } \\
\text { grazing }\end{array}$ & $\begin{array}{l}\text { Found on pebbly and } \\
\text { gravely substrate with } \\
\text { sandy substratum }\end{array}$ & $\begin{array}{l}\text { Rare } \\
\text { Rare and } \\
\text { Endemic } \\
\text { Vulnerable }\end{array}$ & $\begin{array}{l}\text { Nayar \& Sastry } 1988 \\
\text { WCMC } 1994 \\
\text { Rao,K.S.S } 1981 \\
\text { CREB-GUIDE } 2002\end{array}$ \\
\hline $\begin{array}{l}\text { Ipomoea kotschyana } \\
\text { Hoc. ex Choisy }\end{array}$ & Convolvulaceae & 440 & $\begin{array}{l}\text { Thorn mixed } \\
\text { forest }\end{array}$ & $\begin{array}{l}\text { Sand } \\
\text { mining }\end{array}$ & $\begin{array}{l}\text { Sandy substratum and site } \\
\text { specific }\end{array}$ & $\begin{array}{l}\text { Common } \\
\text { Not Evaluated } \\
\text { Indeterminate } \\
\text { Common } \\
\end{array}$ & $\begin{array}{l}\text { Rao } 1981 \\
- \\
\text { WCMC } 1994 \\
\text { CREB-GUIDE } 2002 \\
\end{array}$ \\
\hline $\begin{array}{l}\text { Pavonia ceratocarpa } \\
\text { Mast. }\end{array}$ & Malvaceae & 19 & $\begin{array}{l}\text { Euphorbia } \\
\text { scrub }\end{array}$ & - & $\begin{array}{l}\text { Loamy soil with moderate } \\
\text { soil depth }\end{array}$ & $\begin{array}{l}\text { Rare } \\
\text { Indeterminate } \\
\text { Endangered }\end{array}$ & $\begin{array}{l}\text { Rao } 1981 \\
\text { WCMC } 1994 \\
\text { CREB-GUIDE } 2002\end{array}$ \\
\hline Sida tiagii Bhandari & Malvaceae & 37 & $\begin{array}{l}\text { Acacia } \\
\text { senegal } \\
\text { forest }\end{array}$ & Habitat loss & $\begin{array}{l}\text { Found mainly on open } \\
\text { sandy ground with sparse } \\
\text { vegetation cover }\end{array}$ & $\begin{array}{l}\text { Common } \\
\text { Indeterminate } \\
\text { Common }\end{array}$ & $\begin{array}{l}\text { CREB-GUIDE } 2002 \\
\text { WCMC } 1994 \\
\text { CREB-GUIDE } 2002\end{array}$ \\
\hline $\begin{array}{l}\text { Tribulus } \\
\text { rajasthanensis } \\
\text { Bhandari et Sharma }\end{array}$ & Zygophyllaceae & 43 & $\begin{array}{l}\text { Euphorbia } \\
\text { scrub }\end{array}$ & $\begin{array}{l}\text { Habitat } \\
\text { degradation }\end{array}$ & $\begin{array}{l}\text { Rocky plateau and } \\
\text { sandstone hills }\end{array}$ & $\begin{array}{l}\text { Rare } \\
\text { Indeterminate } \\
\text { Endangered }\end{array}$ & $\begin{array}{l}\text { IUCN } \\
\text { WCMC } 1994 \\
\text { CREB-GUIDE } 2002\end{array}$ \\
\hline
\end{tabular}

* - Wild gymnosperm species

(de Lange \& Norton 2004). Many species at risk of extinction in the United States are declining because of habitat loss and degradation (Hodges \& Elder 2008). Selective cutting causes microclimatic changes and decreases the amount of old and dead trees, which may threaten the persistence of many threatened species (Pykala 2007).

In case of Campylanthus ramosissimus, the common and major threat faced was browsing by cattle, goats and sheep; habitat degradation and some individuals by termites. For Citrullus colocynthis, soil collection in dry riverine/nallahs and naturally dry conditions are prominent threats. Commiphora wightii is threatened due to its illegal exploitation by pharmaceutical and perfumery industries (Sabnis \& Rao 1983). It is also used as folk medicine and is one of the highly commercially exploited species. Poor techniques associated with tapping of gum resin have lead to its total destruction in its natural habitat (Cooke 1958; Kumar \& Bhandari 1994). During this study it was 
Table 2. Distribution of rare, endangered and threatened plants in various habitats in THR

\begin{tabular}{|c|c|c|c|c|c|c|c|c|c|c|c|c|c|c|c|c|c|c|}
\hline \multirow[b]{2}{*}{ Ret plants } & \multirow[b]{2}{*}{$\begin{array}{l}\frac{n}{0} \\
\frac{0}{0} \\
\frac{\pi}{\pi} \\
0 \\
0\end{array}$} & \multicolumn{16}{|c|}{ RET plants in various macro habitat } & \multirow[b]{2}{*}{$\begin{array}{l}\frac{d}{U} \\
\frac{\delta}{ \pm} \\
\frac{0}{0} \\
\frac{\pi}{\pi} \\
\stackrel{0}{0}\end{array}$} \\
\hline & & 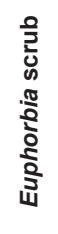 & $\frac{\circ}{\stackrel{\circ}{\alpha}}$ & 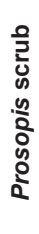 & $\frac{\stackrel{\circ}{\Phi}}{\not}$ & 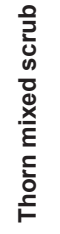 & $\frac{\stackrel{\circ}{\Phi ల ~}}{\propto}$ & $\begin{array}{l}\text { 을 } \\
\text { U. } \\
0 \\
\frac{1}{0} \\
0 \\
0\end{array}$ & $\frac{\circ}{\stackrel{\circ}{0}}$ & 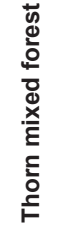 & $\frac{\circ}{\stackrel{\circ}{0}}$ & 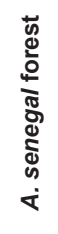 & $\frac{\circ}{\not ்}$ & 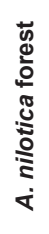 & $\frac{\stackrel{0}{0}}{\not{\alpha}}$ & 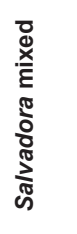 & $\frac{\circ}{\stackrel{\circ}{\alpha}}$ & \\
\hline $\begin{array}{l}\text { Campylanthus } \\
\text { ramosissimus }\end{array}$ & \multirow{13}{*}{ 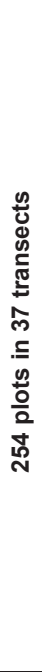 } & 63 & 26.81 & 13 & 5.53 & 27 & 11.49 & 132 & 56.17 & 0 & 0.00 & 0 & 0.00 & 0 & 0.00 & 0.00 & 0.00 & 235 \\
\hline Citrullus colocynthis & & 0 & 0.00 & 3 & 4.62 & 11 & 16.92 & 3 & 4.62 & 17 & 26.15 & 16 & 24.62 & 3 & 4.62 & 12.00 & 18.46 & 65 \\
\hline Commiphora wightii & & 59 & 9.64 & 7 & 1.14 & 192 & 31.37 & 123 & 20.10 & 77 & 12.58 & 112 & 18.30 & 34 & 5.56 & 8.00 & 1.31 & 612 \\
\hline Convolvulus stocksii & & 8 & 6.30 & 0 & 0.00 & 18 & 14.17 & 17 & 13.39 & 57 & 44.88 & 27 & 21.26 & 0 & 0.00 & 0.00 & 0.00 & 127 \\
\hline Dactyliandra we/witschii & & 0 & 0.00 & 0 & 0.00 & 4 & 30.77 & 0 & 0.00 & 6 & 46.15 & 0 & 0.00 & 0 & 0.00 & 3.00 & 23.08 & 13 \\
\hline Dipcadi erythraeum & & 4 & 44.44 & 0 & 0.00 & 0 & 0.00 & 5 & 55.56 & 0 & 0.00 & 0 & 0.00 & 0 & 0.00 & 0.00 & 0.00 & 9 \\
\hline Ephedra foliata & & 4 & 7.27 & 0 & 0.00 & 7 & 12.73 & 4 & 7.27 & 25 & 45.45 & 13 & 23.64 & 2 & 3.64 & 0.00 & 0.00 & 55 \\
\hline Helichrysum cutchicum & & 34 & 13.88 & 15 & 6.12 & 22 & 8.98 & 45 & 18.37 & 32 & 13.06 & 74 & 30.20 & 23 & 9.39 & 0.00 & 0.00 & 245 \\
\hline $\begin{array}{l}\text { Indigofera caerulea var. } \\
\text { monosperma }\end{array}$ & & 0 & 0.00 & 0 & 0.00 & 3 & 18.75 & 6 & 37.50 & 6 & 37.50 & 1 & 6.25 & 0 & 0.00 & 0.00 & 0.00 & 16 \\
\hline Ipomoea kotschyana & & 0 & 0.00 & 0 & 0.00 & 0 & 0.00 & 0 & 0.00 & 335 & 76.14 & 65 & 14.77 & 0 & 0.00 & 40.00 & 9.09 & 440 \\
\hline Pavonia ceratocarpa & & 13 & 68.42 & 2 & 10.53 & 0 & 0.00 & 0 & 0.00 & 0 & 0.00 & 4 & 21.05 & 0 & 0.00 & 0.00 & 0.00 & 19 \\
\hline Sida tiagii & & 7 & 18.92 & 0 & 0.00 & 4 & 10.81 & 5 & 13.51 & 5 & 13.51 & 8 & 21.62 & 8 & 21.62 & 0.00 & 0.00 & 37 \\
\hline Tribulus rajasthanensis & & 13 & 30.23 & 10 & 23.26 & 5 & 11.63 & 5 & 11.63 & 0 & 0.00 & 8 & 18.60 & 2 & 4.65 & 0.00 & 0.00 & 43 \\
\hline \multicolumn{2}{|l|}{ Overall total ret plants } & 205 & 10.70 & 50 & 2.61 & 293 & 15.29 & 345 & 18.01 & 560 & 29.23 & 328 & 17.12 & 72 & 3.76 & 63 & 3.29 & 1916 \\
\hline \multicolumn{2}{|c|}{ Total plots in each habitat } & 27 & 10.6 & 65 & 25.6 & 34 & 13.4 & 23 & 9.07 & 71 & 28 & 25 & 9.84 & 6 & 2.36 & 3 & 1.18 & 254 \\
\hline
\end{tabular}
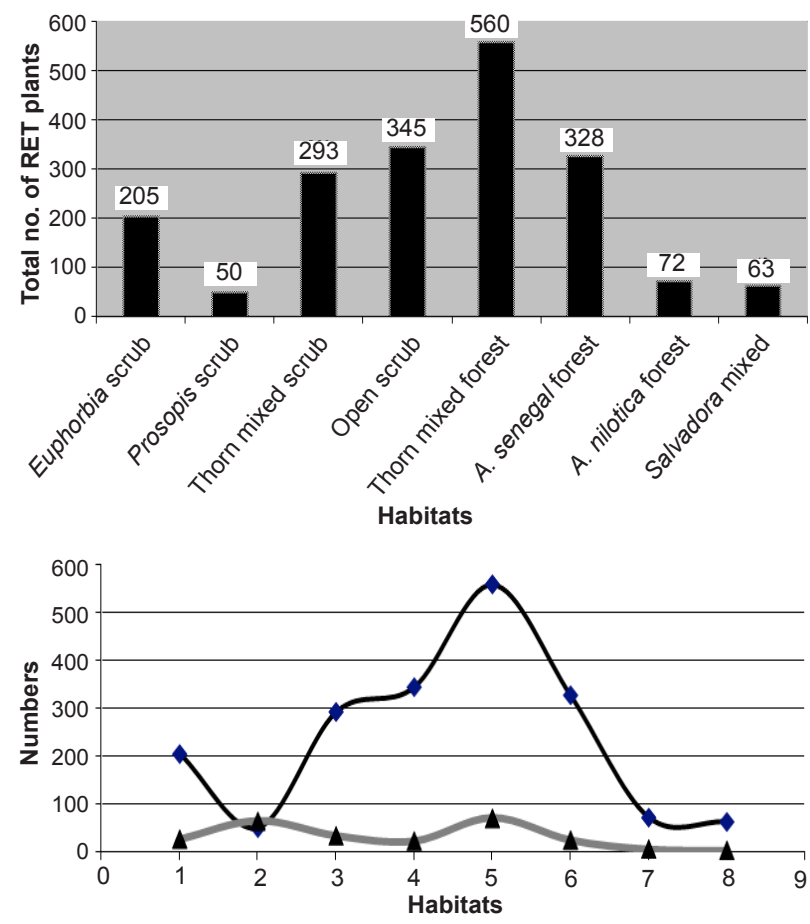

Figure 1. Co-relation between total number of plots and individuals of RET plants in various habitats noted that it was facing four types of anthropogenic and two types of natural threats (Table 4).

Dipcadi erythraeum and Ephedra foliata were threatened by habitat degradation and soil erosion in the study area. No threats were observed on Dactyliandra welwitschii, Indigofera caerulea var. monosperma, Pavonia ceratocarpa and Sida tiagii, during the present investigation, but grazing in the area of occurrence of the species could affect these species by trampling and top soil removal by cattle. In-depth studies are required to identify the threats faced by the species. In the case of Ipomoea kotschyana and Tribulus rajasthanensis, no specific threats were noted, but habitat degradation was observed at a few sites.

The subjective rating of threats based on the field observation showed that except Convolvulus stocksii, all other RET plant taxa faced major threat in the form of habitat degradation (anthropogenic stress or threat) (Table 4; Image 3) due to excessive livestock grazing. 
Table 3. Regeneration status of rare, endangered and threatened (RET) plants

\begin{tabular}{|c|c|c|c|c|c|c|}
\hline \multirow{2}{*}{$\begin{array}{l}\text { Area surveyed } \\
\quad(\ln \mathrm{km} .)\end{array}$} & \multirow{2}{*}{$\begin{array}{l}\text { Sampled units } \\
\text { (In ha) }\end{array}$} & \multirow{2}{*}{$\begin{array}{c}\text { Total adult } \\
\text { plants } \\
(>60 \mathrm{~cm} \text { height) }\end{array}$} & \multirow{2}{*}{$\begin{array}{c}\text { Densityl } \\
\text { ha }\end{array}$} & \multicolumn{3}{|c|}{ Total no. of plants } \\
\hline & & & & $\begin{array}{c}\text { Seedlings } \\
(<10 \mathrm{~cm})\end{array}$ & $\begin{array}{c}\text { Regeneration } \\
(>10-25 \mathrm{~cm})\end{array}$ & $\begin{array}{l}\text { Recruitment } \\
(>25-60 \mathrm{~cm})\end{array}$ \\
\hline \multicolumn{7}{|c|}{ Campylanthus ramosissimus } \\
\hline 5.00 & 1.57 & 32 & 20.36 & $12(37.50 \%)$ & $7(21.87 \%)$ & $2(6.25 \%)$ \\
\hline \multicolumn{7}{|c|}{ Citrullus colocynthis } \\
\hline 2.20 & 0.69 & 9 & 13.02 & $3(33.33 \%)$ & - & - \\
\hline \multicolumn{7}{|c|}{ Commiphora wightii } \\
\hline 12.00 & 3.77 & 286 & 75.84 & $34(11.88 \%)$ & $19(6.64 \%)$ & $13(4.55 \%)$ \\
\hline \multicolumn{7}{|c|}{ Helichrysum cutchicum } \\
\hline 5.00 & 1.57 & 149 & 94.82 & $65(43.63 \%)$ & $38(25.51 \%)$ & $30(2.14 \%)$ \\
\hline
\end{tabular}

\section{DISCUSSION AND CONCLUSIONS}

The phytosociological analysis with ecological information of RET plants revealed that the Campylanthus ramosissimus, Commiphora wightii, Helichrysum cutchicum and Ipomoea kotschyana have abundant populations in the THR. These species require site-specific conservation strategies with the help of the forest department for their long term survival in the study area.

Among the assessed 13 species, five species are reported to be medicinally important in Kachchh (Joshi 2002; Silori et al. 2005; GUIDE 2009). Of these Citrullus colocynthis, Dactyliandra welwitschii, Ephedra foliata and Tribulus rajasthanensis are lightly used, while Commiphora wightii is heavily exploited

Table 4. Information on threats and total counts of RET plant affected

\begin{tabular}{|c|c|c|c|c|c|c|c|c|c|c|c|c|c|c|}
\hline \multirow{3}{*}{ RET plant species } & \multirow{3}{*}{ Total } & \multicolumn{11}{|c|}{ Stress } & \multirow{3}{*}{$\begin{array}{c}\text { Total } \\
\text { Affec- } \\
\text { ted }\end{array}$} & \multirow{3}{*}{$\begin{array}{l}\text { Total } \\
\text { Rel \% }\end{array}$} \\
\hline & & \multicolumn{6}{|c|}{ Anthropogenic } & \multicolumn{5}{|c|}{ Natural } & & \\
\hline & & Cutting & $\begin{array}{l}\text { Brow- } \\
\text { sing }\end{array}$ & $\begin{array}{l}\text { Encroa- } \\
\text { chment }\end{array}$ & $\begin{array}{l}\text { Habitat } \\
\text { Degra- } \\
\text { dation }\end{array}$ & Total & $\begin{array}{c}\text { Rel } \\
\%\end{array}$ & Termites & Dry & Erosion & Total & $\operatorname{Rel} \%$ & & \\
\hline $\begin{array}{l}\text { Campylanthus } \\
\text { ramosissimus }\end{array}$ & 235 & & 27 & & 34 & 61 & 19.37 & 12 & 2 & 7 & 21 & 20.59 & 82 & 19.66 \\
\hline Citrullus colocynthis & 65 & & & & 16 & 16 & 5.08 & & 9 & & 9 & 8.82 & 25 & 6.00 \\
\hline Commiphora wightii & 612 & 54 & 9 & 17 & 20 & 100 & 31.75 & 11 & & 15 & 26 & 25.49 & 126 & 30.22 \\
\hline Convolvulus stocksii & 127 & & 23 & & & 23 & 7.30 & & & & & & 23 & 5.52 \\
\hline $\begin{array}{l}\text { Dactyliandra } \\
\text { we/witschii }\end{array}$ & 13 & & & & & & & & & & & & & \\
\hline Dipcadi erythraeum & 9 & & & & 2 & 2 & 0.63 & & & 2 & 2 & 1.96 & 4 & 0.96 \\
\hline Ephedra foliata & 55 & & & & 4 & 4 & 1.27 & & & 4 & 4 & 3.92 & 8 & 1.92 \\
\hline $\begin{array}{l}\text { Helichrysum } \\
\text { cutchicum }\end{array}$ & 245 & & & & 35 & 35 & 11.11 & & 10 & 30 & 40 & 39.22 & 75 & 17.99 \\
\hline $\begin{array}{l}\text { Indigofera caerulea } \\
\text { var. monosperma }\end{array}$ & 16 & & & & & & & & & & & & & \\
\hline Ipomoea kotschyana & 440 & & & & 70 & 70 & 22.22 & & & & & & 70 & 16.79 \\
\hline Pavonia ceratocarpa & 19 & & & & & & & & & & & & & \\
\hline Sida tiagii & 37 & & & & & & & & & & & & & \\
\hline $\begin{array}{l}\text { Tribulus } \\
\text { rajasthanensis }\end{array}$ & 43 & & & & 4 & 4 & 1.27 & & & & & & 4 & 0.96 \\
\hline Overall total & 1016 & 54 & 59 & 17 & 185 & 315 & 1000 & 23 & 21 & 58 & 102 & 100 & 417 & 1000 \\
\hline Rel \% respectively & 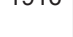 & 17.14 & 18.73 & 5.40 & 58.73 & 100.00 & 100.00 & 22.55 & 20.59 & 56.86 & 100.00 & 100.00 & 21.76 & 100.00 \\
\hline
\end{tabular}




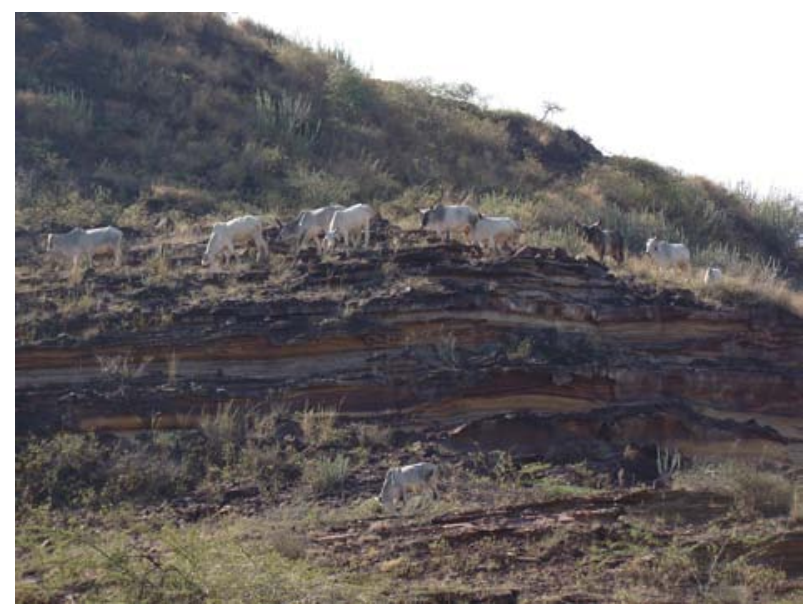

Image 3. RET plants affected through various threats

for local medicine.

It has been reported that a mature $C$. wightii (Guggal), can produce $250-500$ of gum (Atal et al. 1975) and an estimated 300-400 tonnes of Guggal has been sold in Bhuj every year. However, this plant was found to be widely distributed in the study areas as well as in Kachchh. Crude methods of gum extraction from younger plants (Joshi et al. 2004) are likely to affect its abundance in the future. C. wightii is distributed in patches along the study area. This species, being one of the most valuable medicinal plants, needs special attention for its conservation in the wild as well as by promotion through cultivation. Furthermore, this species is endemic to arid and semi-arid regions of the Indian subcontinent (Bole \& Pathak 1988; Dixit \& Rao 2000; GES et al. 2002) and has been listed under promotional programmes of the National Medicinal Plant Board (NMPB), New Delhi. During surveys certain localities such as the site between Sanatorium (2310'39.5” $\left.\mathrm{N} \& 69^{\circ} 38^{\prime} 35.4^{\prime \prime} \mathrm{E}\right)$ and Tapkeshwari Mata Temple in Tapkeshwari MPCAs (Medicinal Plants Conservation Areas) were observed with large patches of $C$. wightii. Likewise, the site between geo-coordinates $23^{\circ} 11^{\prime} 43.6^{\prime \prime} \mathrm{N}$ and $69^{\circ} 25^{\prime} 10.0^{\prime} \mathrm{E}$ to $23^{\circ} 11^{\prime} 37.6^{\prime \prime} \mathrm{N}$ and $69^{\circ} 24^{\prime} 50.3^{\prime} \mathrm{E}$ within THR areas has abundant population of this species. These two sites may be identified for regulated harvesting and seed collection for ex situ conservation of this species by Gujarat State Forest Department.

Awareness of the rarity and the conservation significance of the different species should be created among the locals especially the native healers involved in using these medicinally important RET plants in tradi- tional health care system for healing various types of diseases.

\section{REFERENCES}

Atal, C.K., O.P. Gupta \& S.H. Abag (1975). Commiphora mukul: Sources of Guggal in Indian Systems of Medicine. Economic Botany 29: 208-218.

Bhandari, M.M., D.D. Kaushik \& N.S. Shekhawat (1996). Rare, Threatened and Endangered Plants of the Indian Desert - An Action Plan for their Conservation. Final consolidated report submitted to Department of Biotechnology, Govt. of India, New Delhi, 122pp.

Bhandari, M.M. (1990). Flora of the Indian Desert. Scientific Publishers. Jodhpur, Rajasthan, 435pp.

Bole, P.V. \& J.M. Pathak (1988). The Flora of Saurashtra (Part-II). Botanical Survey of India (BSI), P-8. Brabourne Road Calcutta, 302pp.

Coates, F., I.D. Lunt \& R.L. Tremblay (2006). Effects of disturbance on population dynamics of the threatened orchid Prasophyllum correctum D.L. Jones and implications for grassland management in south-eastern Australia. Biological Conservation 129: 59-69.

Cooke, T. (1958). The Flora of the Presidency of Bombay. Reprinted - Botanical Survey of India, Calcutta, 574pp.

Daniels, R.J.R. \& M. Jayanthi (1996). Biology and conservation of endangered plants: The need to study breeding systems. Tropical Ecology 37(1): 39-42.

de Lange, P.J. \& D.A. Norton (2004). The ecology and conservation of Kunzea sinclairii (Myrtaceae), a naturally rare plant of rhyolitic rock outcrops. Biological Conservation 117: 49-59.

Dixit, A.M. \& S.V.S. Rao (2000). Observation on distribution and habitat characteristics of Gugal (Commiphora wightii) in the arid region of Kachch,, Gujarat (India). Tropical Ecology 41 (1): 81-88.

GES, MSU \& GUIDE (2002). Conservation of rare and endangered biodiversity of Gujarat. Final Project Report submitted to Gujarat Ecology Commission, Vadodara, 428pp.

GUIDE (2009). Establishment of Medicinal Plants Conservation Areas (MPCAs) of Highly Traded and Rare Medicinal Species in Kachchh Saline Desert. Gujarat Institute of Desert Ecology (GUIDE); Bhuj-Kachchh (Gujarat), India, 107pp.

Joshi, P.N. (2002). Study of Ethnobotanical Angiosperms of Bhuj and Mandvi Talukas of Kachchh, Gujarat. PhD Thesis. Department of Botany, Bhavnagar University, Bhavnagar, 341pp.

Joshi, P.N., J. Joshua \& S.F.W. Sunderraj (2004). Population structure and dynamics of threatened plant species in Bhuj and Mandvi Talukas of Kachchh District. Advances in Biological Sciences 3: 13-17.

Kumar, A. \& M.M. Bhandari (1994). Commiphora wightii 
-A threatened medicinal plant of the Thar Pradesh. In: Etnobiology in Human Welfare. Abstracts of the 4th International congress of Ethnobiology, Lucknow, Uttar Pradesh, India, 17-21 November, 307pp.

Nayar, M.P. \& A.R.K. Sastry (1988). Red Data Book of Indian Plants-Vol. 2. Botanical Survey of India. Calcutta, 271pp.

Pykala, J. (2007). Implementation of Forest Act habitats in Finland: Does it protect the right habitats for threatened species? Forest Ecology and Management 242: 281-287.

Rao, K.S.S. (1981). Studies on the flora of South Eastern Kutch. Ph.D Thesis. M.S. University, Vadodara.

Raole, V.M. (1993). Studies on endangered and endemic desert taxa. PhD Thesis. Department of Botany, M.S. University, Vadodara.

Sabnis, S.D. \& K.S.S. Rao (1983). Observation on some rare and endangered endemics of south eastern Kachchh, pp. 71-77. In: Jain, S.K. \& R.R. Rao (eds.). Assessment of Threatened Plants of India. Botanical Survey of India, Howrah.

Shah, G.L. (1978). Flora of Gujarat State. University Press, Sardar Patel University. Vallabh Vidyanagar, 1074pp.

Shaw, W.B. \& B.R. Burns (1997). The ecology and conservation of the endangered endemic shrub, Kowhai Ngutukaka Clianthus puniceus in New Zealand. Biological Conservation 81: 233-245.

Silori, C.S., A.M. Dixit, L. Gupta \& N. Mistry (2005). Observation on medicinal plant richness and associated conservation issues in district Kachchh, Gujarat, Trivedi, P.C. (ed.). In: Medicinal Plants: Utilization and Conservation. Rajasthan University, Rajasthan.

Tian, Z., C. Weilie, Z. Changming, C. Yue \& Z. Binghui (2007). Plant biodiversity and its conservation strategy in the inundation and resettlement districts of the Yangtze Three Gorges, China. Acta Ecologica Sinica 27(8): 3110-3118.

WCMC (1994). Status report as of 24 November 1994, Gujarat, Printout from plant database BG- BASE. World Conservation Monitoring Centre.

Zietsman, J., L.L. Dreyer \& K.J. Esler (2008). Reproductive biology and ecology of selected rare and endangered Oxalis L. (Oxalidaceae) plant species. Biological Conservation 141: 1475-1483.
Author Details: DR. P.N. JosH has a research experience of 12 years and published 48 research articles on plant ecology, participatory natural resource management and conservation. He is the registered member of IUCN - The World Conservation Union: Species Survival Commission Indian Subcontinent Plant Specialist Group (SSC-ISPSG) and Indian Association for Angiosperm Taxonomy (IAAT). DR. EkTA B. Joshi, has a PhD in Plant Science (Ecology, Taxonomy and Conservation). She has research experience of five years and published eight articles in the fields of plant taxonomy, conservation of rare and endangered plants, ethnobotany among others.

DR. B.K. JaIn, Principal in M.G.Science Institute, Ahmedabad and has a research and teaching experience of more than 20 years. He has published several books on vegetation science and is doing research in various branches of botany. 